\title{
Prognostic Association of Circulating Neutrophil Count with No-Reflow in Patients with ST-Segment Elevation Myocardial Infarction following Successful Primary Percutaneous Intervention
}

\author{
Jinfan Tian, ${ }^{1,2}$ Yue Liu, ${ }^{3}$ Yanfei Liu, ${ }^{4}$ Xiantao Song, ${ }^{1,2}$ Min Zhang, ${ }^{1,2}$ Feng Xu, ${ }^{1,2}$ Fei Yuan, \\ and Shuzheng Lyu ${ }^{1,2}$ \\ ${ }^{1}$ Department of Cardiology, Beijing Anzhen Hospital, Capital Medical University, Beijing 100029, China \\ ${ }^{2}$ Beijing Institute of Heart, Lung and Blood Vessel Diseases, Beijing 100029, China \\ ${ }^{3}$ Cardiovascular Disease Centre of Xiyuan Hospital, China Academy of Chinese Medical Sciences, Beijing 100091, China \\ ${ }^{4}$ Graduate School, Beijing University of Chinese Medicine, Beijing 100029, China \\ Correspondence should be addressed to Shuzheng Lyu; shuzheng@medmail.com.cn
}

Received 27 June 2017; Revised 30 September 2017; Accepted 31 October 2017; Published 12 December 2017

Academic Editor: Zhongjie Shi

Copyright (c) 2017 Jinfan Tian et al. This is an open access article distributed under the Creative Commons Attribution License, which permits unrestricted use, distribution, and reproduction in any medium, provided the original work is properly cited.

\begin{abstract}
Objective. The aim of the present study was to investigate the predictive value of neutrophil count for no-reflow in patients with ST-segment elevation myocardial infarction (STEMI) who underwent successful primary percutaneous intervention (PCI). Methods. We conducted a retrospective study of 361 patients diagnosed with acute STEMI between 2011 and 2015. All patients underwent successful PCI within $12 \mathrm{~h}$ from the onset of symptoms. Angiographic no-reflow was diagnosed based on a post-PCI thrombolysis in myocardial infarction flow grade $\leq 2$ without mechanical obstruction. According to a neutrophil count cut-off determined by receiver operating characteristic curve analysis, patients were divided into two groups: group A (neutrophil count $<9.14 \times 10^{9} / \mathrm{L}$ ) and group B (neutrophil count $\geq 9.14 \times 10^{9} / \mathrm{L}$ ). Results. Compared to patients in the normal reflow group, patients with no-reflow had higher neutrophil counts $(P<0.05)$. The incidence rate of no-reflow in group A $(18,9.3 \%)$ was significantly lower than that in group B (38). Multivariate logistic regression analysis revealed that a neutrophil count $\geq 9.14 \times 10^{9} / \mathrm{L}$ was independently predictive for no-reflow (odds ratio $=4.474,95 \%$ confidence interval: $1.610-12.433, P=0.004)$ after adjusting for potential confounders. Conclusions. A circulating neutrophil count $\geq 9.14 \times 10^{9} / \mathrm{L}$ is independently associated with no-reflow in patients with acute STEMI following primary PCI.
\end{abstract}

\section{Introduction}

ST-segment elevation myocardial infarction (STEMI) is known to be one of the leading causes of mortality worldwide. Primary percutaneous coronary intervention (PCI) is the most effective way of preventing the progression of myocardial necrosis and reducing mortality associated with STEMI. However, according to Kloner et al. [1] under some circumstance, restoration of arterial flow into the previously ischemic tissue either does not occur or is greatly impeded. Early and adequate restoration of the infarct-related artery (IRA) does not always result in optimal myocardial reperfusion [2]. This phenomenon is defined as "no-reflow" $[1,2]$. No-reflow reduces the benefits of primary PCI in patients with acute STEMI. The size of the "no-reflow" zone is closely correlated with cardiac systolic function, myocardial remodeling, ventricular arrhythmias, cardiogenic shock, mortality during hospitalization, and worsened outcomes at 
follow-up [3]. Currently, no single effective therapeutic approach is available for no-reflow, making prevention vital. Identifying patients at the greatest risk is the first step in the prevention of no-reflow [4]. It is necessary to detect available blood biomarkers and other clinical indices to predict the risk of no-reflow and reduce the incidence of this phenomenon in the early stage.

Myocardial ischemia/reperfusion injury is the most important pathological characteristic in the development of no-reflow [5]. Recent fundamental studies have shown that neutrophils become trapped in an area of myocardial ischemia reperfusion via the NF- $\kappa \mathrm{B}$ cascade [6-9]. Trapped leukocytes are established as important inflammation mediators of cardiac ischemia/reperfusion injury [8, 10-12]. Furthermore, clinical studies have reported that neutrophil accumulation at the coronary culprit lesion site predicts mortality in patients with acute coronary syndrome (ACS)/acute myocardial infarction (AMI) [13]. We hypothesized that the trapped neutrophils are derived from the circulating blood in the context of ischemiareperfusion. With the above in mind, the aim of the present study was to investigate the prognostic association of an easily detectable biomarker-the count of circulating neutrophils with angiographic no-reflow assessed by postPCI thrombolysis in myocardial infarction (TIMI) flow grade. Therefore, the neutrophil count on admission was considered in the present study.

\section{Methods}

2.1. Patients. A total of 361 patients diagnosed with acute STEMI from January 2011 to December 2015 were enrolled retrospectively in the present study. All patients underwent successful primary PCI within 12 hours from the onset of symptoms. The Ethics Committee of Anzhen Hospital approved the study protocol (Beijing, China).

2.2. STEMI Diagnostic Criteria. The STEMI diagnostic criteria are as follows: (1) typical ischemic chest pain lasting for at least $30 \mathrm{~min}$ and not alleviated by resting or nitroglycerin; (2) ST-segment elevation $\geq 2 \mathrm{~mm}$ in at least two consecutive leads or the onset of left bundle branch block; and (3) an increase and/or a decrease in cardiac biomarker values (preferably troponin), with at least one value above the 99th percentile of the upper reference limit [14].

2.3. Inclusion Criteria. The inclusion criteria are as follows: (1) patients diagnosed with STEMI; (2) men and nonpregnant women between 18 and 80 years of age; and (3) patients who signed the informed consent forms.

2.4. Exclusion Criteria. The exclusion criteria are as follows: (1) patients with cardiac shock; (2) patients with valvular heart disease; (3) patients with cardiomyopathy; (4) patients who underwent coronary artery bypass grafting; (5) heart transplant recipients; (6) patients with contraindications to antiplatelet agents and anticoagulation; (7) patients with multiple organ failure; (8) patients with acute infection, autoimmune disorders, or advanced cancer; and (9) patients allergic to contrast agents. All patients were divided into the no-reflow group and normal reflow group according to TIMI flow grade during coronary angiography (as illustrated below).

2.5. Diagnosis of No-Reflow during Coronary Angiography. All patients were administered with oral aspirin $(300 \mathrm{mg})$ and clopidogrel $(300 \mathrm{mg})$ and intravenous unfractionated heparin (50-70 U/kg). PCI procedures were performed via the transradial and transfemoral approaches. Before PCI, each patient underwent left and right coronary angiography with at least two projections. The upfront intracoronary administration of glycoprotein IIb/IIIa receptor inhibitor (GPIIb/IIIa receptor inhibitor, Tirofiban) was left to the operator's discretion during PCI. After intervention, all patients were administered with clopidogrel $(75 \mathrm{mg})$ and aspirin (100 mg) once daily for 12 months. Other treatments were provided according to the physician's clinical opinion.

At least two experienced cardiologists determined all parameters and strategies. No-reflow was defined as a postPCI TIMI flow grade of $\leq 2$ in the IRA in the absence of dissection, spasm, apparent thrombus, or flow-limiting residual stenosis $<50 \%$. TIMI flow grade 3 was considered as normal reflow [15-17].

2.6. Grouping. Based on the neutrophil count cut-off determined by the receiver operating characteristic (ROC) curve analysis, the patients were divided into two groups: group A (neutrophil count $<9.14 \times 10^{9} / \mathrm{L}$ ) and group B (neutrophil count $\left.\geq 9.14 \times 10^{9} / \mathrm{L}\right)$, namely, group A $(<9.4 \mathrm{G} / \mathrm{L})$ and group $\mathrm{B}(\geq 9.4 \mathrm{G} / \mathrm{L})$.

2.7. Laboratory Analysis. Blood samples were drawn from the antecubital vein on admission for laboratory analysis. Neutrophil count was determined from the whole blood using an automated haematology analyser. Samples were centrifuged within $30 \mathrm{~min}$ to separate plasma and to determine the serum creatinine (Scr), blood glucose (GLU), and blood lipid profiles (low-density lipoprotein, high-density lipoprotein cholesterol, total cholesterol, and triglycerides) using an automated biochemical analyser. Cardiac biomarkers and high-sensitivity C-reactive protein (Hs-CRP) levels were measured using standard methods.

2.8. Clinical Data Collection and Quality Control. Responsible physicians performed the physical examinations and the independent researchers recorded data related to smoking history and comorbidities (diabetes mellitus and hypertension) on admission. The Killip classification was used to assess the severity of heart failure. Primary PCI strategy, choice of stent, and medications administered during hospitalization were chosen by the individual interventional cardiologists or responsible physicians according to clinical symptoms and angiographic characteristics. Data used for statistical analysis were obtained and entered into a computerized database by the staff.

2.9. Statistical Analysis. Continuous variables were presented as means \pm standard deviations. When the variables were normally distributed, Student's $t$-test was used to compare 
two independent samples. The Mann-Whitney $U$ test was used to compare nonnormally distributed data. Categorical variables were expressed as frequencies and percentages, and the chi-square test was used to compare the data. An ROC curve was used to determine neutrophil count cut-off level. The predictors of no-reflow were determined by univariate and multivariate logistic regression. In multivariate models, covariates included age $\geq 65$, male, smoking history, hypertension, diabetes, Killip classification $\geq 3$, the left anterior descending artery (LAD) as the IRA, neutrophil count $\geq 9.14 \times 10^{9} / \mathrm{L}$, neutrophil/lymphocyte ratio, cardiac troponin I (cTNI), upfront intracoronary GPIIb/IIIa receptor inhibitor administration, aspiration thrombectomy, platelet counts, white blood cell (WBC) counts, hemoglobin (HGB), time from symptom onset to reperfusion ( $>6$ hours), multivessel disease, and initial TIMI flow grade (0-1) (those with a $P$ value $<0.1$ in univariate analysis and those that were clinically relevant). Results were presented as adjusted odds ratios (ORs) with 95\% confidence intervals (CIs). A twosided $P$ value $<0.05$ was considered statistically significant. SPSS 17.0 software was used to analyse the data.

\section{Results}

3.1. Baseline Characteristics. ROC curve analysis revealed that neutrophil count predicted no-reflow. The area under the ROC curve was 0.604 (95\% CI: $0.522-0.687, P=0.013$ ) (see Figure 1), and the neutrophil count cut-off value was $9.14 \times 10^{9} / \mathrm{L}$, with $67.9 \%$ sensitivity and $57.7 \%$ specificity. Patients were divided into two groups according to the neutrophil count cut-off level: group A $(<9.4 \mathrm{G} / \mathrm{L})(n=194)$ and group $\mathrm{B}(\geq 9.4 \mathrm{G} / \mathrm{L})(n=167)$.

The mean age in group $A(<9.4 \mathrm{G} / \mathrm{L})$ was greater than that in group $B(\geq 9.4 \mathrm{G} / \mathrm{L})$. Differences in gender, smoking history, hypertension, diabetes, and history of PCI between the two groups were not statistically significant. No statistically significant differences were found between group A $(<9.4 \mathrm{G} / \mathrm{L})$ and group $\mathrm{B}(\geq 9.4 \mathrm{G} / \mathrm{L})$ in terms of blood pressure, time from onset of symptoms to reperfusion, and multivessel disease. There were $53(17.3 \%)$ patients with Killip class I in group A $(<9.4 \mathrm{G} / \mathrm{L})$ compared to $35(21.1 \%)$ in group $B(\geq 9.4 \mathrm{G} / \mathrm{L})$. There were $128(66.0 \%)$ patients with Killip class II in group A $(<9.4 \mathrm{G} / \mathrm{L})$ compared to $119(71.7 \%)$ in group $B(\geq 9.4 \mathrm{G} / \mathrm{L})$. There were $11(5.7 \%)$ patients with Killip class III in group A $(<9.4 \mathrm{G} / \mathrm{L})$ compared to eight $(4.8 \%)$ in group B $(\geq 9.4 \mathrm{G} / \mathrm{L})$. There were $2(1.0 \%)$ patients with Killip class IV in group A $(<9.4 \mathrm{G} / \mathrm{L})$ compared to four $(2.4 \%)$ in group $\mathrm{B}(\geq 9.4 \mathrm{G} / \mathrm{L})$. There were more patients with multistent implantation in group $A(<9.4 \mathrm{G} / \mathrm{L})(68$, $35.1 \%)$ than in group B $(\geq 9.4 \mathrm{G} / \mathrm{L})(41,24.6 \%)$. Higher WBC counts, neutrophil/lymphocyte ratios, red blood cell counts, HGB, platelet counts, and cTNI were detected in group $B(\geq 9.4 \mathrm{G} / \mathrm{L})$, whereas the lymphocyte counts and proportion of lymphocytes were decreased correspondingly. The differences in other blood markers were not statistically significant (see Table 1).

3.2. The Incidence of No-Reflow in Group $A(<9.4 \mathrm{G} / \mathrm{L})$ and Group $B(\geq 9.4 G / L)$. Eighteen $(9.3 \%)$ patients in group A

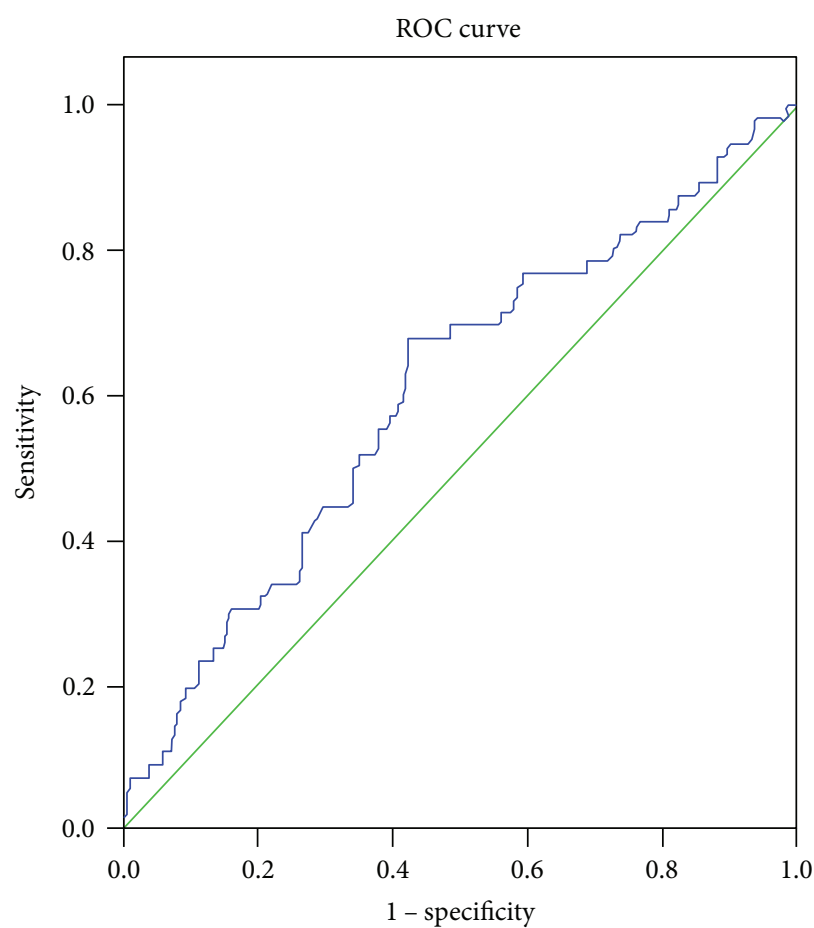

FIGURE 1: Receiver operating characteristic analysis of neutrophil count and no-reflow (area under the curve 0.604, 95\% confidence interval: $0.522-0.687, P=0.013)$.

$(<9.4 \mathrm{G} / \mathrm{L})$ had no-reflow compared to 38 (22.8\%) patients in group $B(\geq 9.4 \mathrm{G} / \mathrm{L})$. This difference was statistically significant (Table 2).

3.3. Clinical Characteristics of Patients in the Normal Reflow Group and No-Reflow Group. No differences between patients in the no-reflow group and normal reflow group were detected in terms of age, gender, smoking history, comorbidities, history of PCI, and blood pressure. Higher values of WBC counts, neutrophil counts, neutrophil proportions, and neutrophil to lymphocyte ratio were detected in the noreflow group compared to those in the normal reflow group. Lymphocyte counts and the proportion of lymphocytes were lower in the no-reflow group than those in the normal reflow group. Blood lipids, GLU, and other blood markers were not statistically significantly different between the two groups. The differences between the two groups with respect to left ventricular diastolic dysfunction and ejection fraction on admission were not statistically significant. Patients with no-reflow had significantly higher Killip classifications than those with normal reflow $(P<0.05)$ (Table 3$)$.

3.4. Coronary Angiography Findings and Percutaneous Intervention Characteristics in the No-Reflow and Normal Reflow Groups. No-reflow, defined according to TIMI flow grade during coronary angiography, was more frequently observed among patients with the LAD as the IRA, while TIMI flow grade 3 was more frequently observed within the right coronary artery (RCA). There were more patients with anterior wall infarction in the no-reflow group than in the normal reflow group. The upfront intracoronary GPIIb/IIIa 
TABLE 1: Baseline characteristics in group A and group B.

\begin{tabular}{|c|c|c|c|}
\hline Parameters & Group A $(<9.4 \mathrm{G} / \mathrm{L})(n=194)$ & Group B $(\geq 9.4 \mathrm{G} / \mathrm{L})(n=167)$ & $P$ value \\
\hline Age (years, $\bar{X} \pm S$ ) & $56.48 \pm 10.85$ & $53.57 \pm 10.86$ & $0.012^{*}$ \\
\hline Gender, male, $n(\%)$ & $171(88.1)$ & $148(88.6)$ & 0.888 \\
\hline Smoker, $n(\%)$ & $129(66.5)$ & $120(71.9)$ & 0.272 \\
\hline Hypertension, $n(\%)$ & $105(54.1)$ & $90(53.9)$ & 0.965 \\
\hline Diabetes, $n(\%)$ & $50(25.8)$ & $44(26.3)$ & 0.901 \\
\hline History of PCI, $n(\%)$ & $12(6.2)$ & $7(4.2)$ & 0.398 \\
\hline $\mathrm{SBP}(\mathrm{mmHg}, \bar{X} \pm \mathrm{S})$ & $120.12 \pm 17.93$ & $116.60 \pm 17.11$ & 0.074 \\
\hline $\mathrm{DBP}(\mathrm{mmHg}, \bar{X} \pm \mathrm{S})$ & $74.93 \pm 10.94$ & $73.82 \pm 11.40$ & 0.579 \\
\hline Time from onset of symptoms to reperfusion (hour) & $5.19 \pm 2.72$ & $5.25 \pm 2.44$ & 0.443 \\
\hline Killip classification, $n(\%)$ & & & 0.395 \\
\hline 1 & $53(17.3)$ & $35(21.1)$ & \\
\hline 2 & $128(66.0)$ & $119(71.7)$ & \\
\hline 3 & $11(5.7)$ & $8(4.8)$ & \\
\hline 4 & $2(1.0)$ & $4(2.4)$ & \\
\hline WBC counts $\left(\times 10^{9} / \mathrm{L}\right)$ & $8.83 \pm 1.69$ & $13.83 \pm 2.62$ & $0.0001^{* *}$ \\
\hline Lymphocyte counts $\left(\times 10^{9} / \mathrm{L}\right)$ & $1.65 \pm 0.89$ & $1.46 \pm 0.81$ & $0.014^{*}$ \\
\hline Proportion of lymphocytes (\%) & $18.84 \pm 9.04$ & $10.70 \pm 5.25$ & $0.0001^{* *}$ \\
\hline Neutrophil/lymphocyte ratio & $5.38 \pm 4.12$ & $9.89 \pm 4.88$ & $0.0001^{* *}$ \\
\hline Red blood cell counts $\left(\times 10^{12} / \mathrm{L}\right)$ & $4.56 \pm 0.48$ & $4.76 \pm 0.57$ & $0.0001^{* *}$ \\
\hline HGB (g/L) & $141.45 \pm 14.33$ & $145.72 \pm 14.84$ & $0.006^{* *}$ \\
\hline Platelet counts $\left(\times 10^{9} / \mathrm{L}\right)$ & $202.65 \pm 55.48$ & $221.62 \pm 71.07$ & $0.001^{* *}$ \\
\hline PDW (\%) & $12.15 \pm 1.85$ & $12.46 \pm 1.82$ & 0.115 \\
\hline cTNI (ng/L) & $65.64 \pm 55.83$ & $90.63 \pm 71.91$ & $0.0001^{* *}$ \\
\hline $\operatorname{Scr}(\mu \mathrm{mol} / \mathrm{L})$ & $76.99 \pm 18.63$ & $79.37 \pm 23.36$ & 0.489 \\
\hline $\mathrm{TG}(\mathrm{mmol} / \mathrm{L})$ & $1.86 \pm 1.50$ & $1.84 \pm 1.01$ & 0.419 \\
\hline TCHO (mmol/L) & $4.62 \pm 0.89$ & $4.65 \pm 0.98$ & 0.811 \\
\hline HDL-C (mmol/L) & $1.02 \pm 0.23$ & $0.99 \pm 0.23$ & 0.246 \\
\hline LDL-C (mmol/L) & $2.91 \pm 0.78$ & $3.02 \pm 0.87$ & 0.245 \\
\hline GLU (mmol/L) & $7.88 \pm 2.59$ & $8.43 \pm 3.07$ & 0.129 \\
\hline Hs-CRP (mg/L) & $9.54 \pm 10.14$ & $11.75 \pm 10.47$ & 0.051 \\
\hline FBG $(g / L)$ & $2.72 \pm 0.56$ & $2.72 \pm 0.71$ & 0.985 \\
\hline IRA, $n(\%)$ & & & 0.802 \\
\hline LAD & $95(49.0)$ & $79(47.3)$ & \\
\hline LCX & $18(9.3)$ & $19(11.4)$ & \\
\hline RCA & $81(41.8)$ & $69(41.3)$ & \\
\hline Multivessel disease, $n(\%)$ & $48(24.7)$ & $42(25.1)$ & 0.929 \\
\hline Multistent, $n(\%)$ & $68(35.1)$ & $41(24.6)$ & $0.030^{*}$ \\
\hline
\end{tabular}

${ }^{*} P<0.05 ;{ }^{* *} P<0.01$. SBP: systolic blood pressure; DBP: diastolic blood pressure; WBC: white blood cell; HGB: hemoglobin; PDW: platelet distribution width; cTNI: cardiac troponin I; Scr: serum creatinine; TG: triglyceride; TCHO: total cholesterol; HDL-C: high-density lipoprotein cholesterol; LDL-C: low-density lipoprotein cholesterol; GLU: glucose; Hs-CRP: high-sensitivity C-reactive protein; FBG: fibrinogen; IRA: infarct-related artery; LAD: left anterior descending; LCX: left circumflex artery; RCA: right coronary artery.

TABLE 2: The incidence of no-reflow in group A and group B.

\begin{tabular}{lccc}
\hline Parameters & $\begin{array}{c}\text { Group A } \\
(<9.4 \mathrm{G} / \mathrm{L}) \\
(n=194)\end{array}$ & $\begin{array}{c}\text { Group B } \\
(\geq 9.4 \mathrm{G} / \mathrm{L}) \\
(n=167)\end{array}$ & $P$ value \\
\hline No-reflow, $n(\%)$ & & & $0.0001^{* *}$ \\
Yes & $18(9.3)$ & $38(22.8)$ & \\
No & $176(90.7)$ & $129(77.2)$ & \\
\hline$* * P<0.01$ & & &
\end{tabular}

${ }^{* *} P<0.01$. receptor inhibitor use rate was lower in the no-reflow group than the normal flow group. However, the incidence of no-reflow was not affected by multivessel disease, multistent implantation, and aspiration thrombectomy. Moreover, there were no significant differences between the no-reflow and normal reflow groups with respect to time from symptom onset to reperfusion and initial TIMI flow grade. Non-IRA intervention was not associated with no-reflow (Table 4). 
TABLE 3: Clinical characteristics of patients with normal reflow and no-reflow.

\begin{tabular}{|c|c|c|c|}
\hline Parameters & Normal reflow $(n=305)$ & No-reflow $(n=56)$ & $P$ value \\
\hline Age (years, $\bar{X} \pm S$ ) & $54.96 \pm 11.80$ & $56.11 \pm 11.66$ & 0.470 \\
\hline Gender, male, $n(\%)$ & $269(88.2)$ & $50(89.3)$ & 0.815 \\
\hline Smoker, $n(\%)$ & $212(69.5)$ & $37(66.1)$ & 0.609 \\
\hline Hypertension, $n(\%)$ & $165(54.1)$ & $30(53.6)$ & 0.942 \\
\hline Diabetes, $n(\%)$ & $80(26.2)$ & $14(25.0)$ & 0.847 \\
\hline History of PCI, $n(\%)$ & $18(5.9)$ & $1(1.8)$ & 0.330 \\
\hline $\mathrm{SBP}(\mathrm{mmHg}, \bar{X} \pm \mathrm{S})$ & $118.8 \pm 17.6$ & $116.9 \pm 17.7$ & 0.504 \\
\hline $\mathrm{DBP}(\mathrm{mmHg}, \bar{X} \pm \mathrm{S})$ & $74.4 \pm 11.3$ & $74.6 \pm 10.6$ & 0.983 \\
\hline Killip classification, $n(\%)$ & & & $0.011^{*}$ \\
\hline 1 & $82(26.9)$ & $7(12.5)$ & \\
\hline 2 & $206(67.5)$ & $41(73.2)$ & \\
\hline 3 & $12(3.9)$ & $7(12.5)$ & \\
\hline 4 & $5(1.6)$ & $1(1.8)$ & \\
\hline WBC counts $\left(\times 10^{9} / \mathrm{L}\right)$ & $10.99 \pm 3.23$ & $11.97 \pm 3.61$ & $0.042^{*}$ \\
\hline Proportion of neutrophils (\%) & $78.73 \pm 11.11$ & $82.73 \pm 9.15$ & $0.002^{* *}$ \\
\hline Neutrophil counts $\left(\times 10^{9} / \mathrm{L}\right)$ & $8.79 \pm 3.21$ & $10.01 \pm 3.45$ & $0.013^{*}$ \\
\hline Lymphocyte counts $\left(\times 10^{9} / \mathrm{L}\right)$ & $1.61 \pm 0.89$ & $1.34 \pm 0.45$ & $0.034^{*}$ \\
\hline Proportion of lymphocytes (\%) & $15.59 \pm 8.69$ & $12.29 \pm 7.19$ & $0.002^{* *}$ \\
\hline Neutrophil/lymphocyte ratio & $7.17 \pm 4.93$ & $9.10 \pm 5.19$ & $0.001^{* *}$ \\
\hline Red blood cell counts $\left(\times 10^{12} / \mathrm{L}\right)$ & $4.66 \pm 0.53$ & $4.62 \pm 0.56$ & 0.649 \\
\hline $\mathrm{HGB}(\mathrm{g} / \mathrm{L})$ & $143.71 \pm 14.55$ & $141.88 \pm 15.54$ & 0.391 \\
\hline Platelet counts $\left(\times 10^{9} / \mathrm{L}\right)$ & $212.57 \pm 64.85$ & $205.19 \pm 57.79$ & 0.467 \\
\hline PDW (\%) & $12.27 \pm 1.87$ & $12.44 \pm 1.70$ & 0.529 \\
\hline cTNI (ng/L) & $74.09 \pm 62.49$ & $94.15 \pm 75.05$ & 0.078 \\
\hline $\mathrm{Scr}(\mu \mathrm{mol} / \mathrm{L})$ & $77.66 \pm 21.19$ & $80.45 \pm 19.61$ & 0.155 \\
\hline $\mathrm{TG}(\mathrm{mmol} / \mathrm{L})$ & $1.88 \pm 1.37$ & $1.70 \pm 0.83$ & 0.795 \\
\hline TCHO $(\mathrm{mmol} / \mathrm{L})$ & $4.65 \pm 0.92$ & $4.53 \pm 1.00$ & 0.382 \\
\hline HDL-C (mmol/L) & $1.00 \pm 0.23$ & $1.04 \pm 0.25$ & 0.310 \\
\hline LDL-C (mmol/L) & $2.97 \pm 0.81$ & $2.92 \pm 0.90$ & 0.696 \\
\hline GLU (mmol/L) & $8.17 \pm 2.94$ & $7.95 \pm 2.19$ & 0.717 \\
\hline Hs-CRP (mg/L) & $10.62 \pm 10.48$ & $10.21 \pm 9.49$ & 0.869 \\
\hline FBG $(g / L)$ & $2.73 \pm 0.64$ & $2.64 \pm 0.63$ & 0.574 \\
\hline LVDD (mm) & $49.70 \pm 4.65$ & $49.09 \pm 7.81$ & 0.728 \\
\hline $\mathrm{EF}(\%)$ & $54.30 \pm 9.49$ & $52.91 \pm 10.28$ & 0.355 \\
\hline
\end{tabular}

${ }^{*} P<0.05 ;{ }^{* *} P<0.01$. LVDD: left ventricular diastolic diameter; EF: ejection fraction.

3.5. Univariate and Multivariate Logistic Regression. In univariate analysis, the Killip classification $\geq 3(\mathrm{OR}=2.824,95 \% \mathrm{CI}$ : $1.155-6.904, P=0.023$ ), LAD as the IRA (OR $=1.821,95 \%$ CI: $1.018-3.259, P=0.043)$, neutrophil count $\geq 9.14 \times 10^{9} / \mathrm{L}$ (OR=2.880, 95\% CI: $1.573-5.275, P=0.001)$, neutrophil/ lymphocyte ratio $(\mathrm{OR}=1.067,95 \% \mathrm{CI}: 1.015-1.121, P=$ $0.011)$, cTNI $(\mathrm{OR}=1.004,95 \% \mathrm{CI}: 1.000-1.008, P=0.036)$, and $\mathrm{WBC}$ count $(\mathrm{OR}=1.086,95 \% \mathrm{CI}$ : $1.002-1.178, P=$ $0.046)$ were predictors for no-reflow. Upfront intracoronary GPIIb/IIIa receptor inhibitor use was negatively associated with no-reflow $(\mathrm{OR}=0.303,95 \% \mathrm{CI}$ : 0.091-1.010, $P=0.052$ ). In multivariate logistic regression analysis, neutrophil count $\geq 9.14 \times 10^{9} / \mathrm{L}$ was a predictor for no-reflow after adjusting for patients aged $\geq 65$, male, smoking, hypertension, diabetes, Killip classification $\geq 3$, LAD as the IRA, neutrophil/lymphocyte ratio, cTNI, upfront intracoronary GPIIb/IIIa receptor inhibitor use, aspiration thrombectomy, platelet counts, WBC counts, HGB, time from symptom onset to reperfusion ( $>6$ hours), multivessel disease, and initial TIMI flow grade $(0-1)(\mathrm{OR}=4.474,95 \% \mathrm{CI}: 1.610-$ 12.433, $P=0.004$ ) (Table 5).

\section{Discussion}

In the present study, there were 56 (15.5\%) patients with angiographic no-reflow. Patients in the no-reflow group 
TABLE 4: Percutaneous intervention findings of patients with normal reflow and no-reflow.

\begin{tabular}{|c|c|c|c|}
\hline Parameters & Normal reflow $(n=305)$ & No-reflow $(n=56)$ & $P$ value \\
\hline Time from onset of symptoms to reperfusion (hour) & $5.20 \pm 2.70$ & $5.29 \pm 1.95$ & 0.315 \\
\hline Time from onset of symptoms to reperfusion ( $>6$ hours) & $96(31.5)$ & $16(28.6)$ & 0.666 \\
\hline \multicolumn{4}{|l|}{ Multivessel disease, $n(\%)$} \\
\hline Yes & $76(24.9)$ & $14(25.0)$ & \multirow[t]{2}{*}{0.990} \\
\hline No & $229(75.1)$ & $76(24.9)$ & \\
\hline \multicolumn{4}{|l|}{ Initial TIMI flow grade, $n(\%)$} \\
\hline $0-1$ & $192(63)$ & $38(67.9)$ & \multirow[t]{2}{*}{0.483} \\
\hline$\geq 2$ & $113(37.1)$ & $18(32.1)$ & \\
\hline \multicolumn{4}{|l|}{ Multistent, $n(\%)$} \\
\hline Yes & $93(30.5)$ & $16(28.6)$ & \multirow[t]{2}{*}{0.774} \\
\hline No & $212(69.5)$ & $40(71.4)$ & \\
\hline \multicolumn{4}{|l|}{ IRA, $n(\%)$} \\
\hline LAD & $140(45.9)$ & $34(60.7)$ & \multirow[t]{3}{*}{0.051} \\
\hline LCX & $30(9.8)$ & $7(12.5)$ & \\
\hline RCA & $135(44.3)$ & $15(26.8)$ & \\
\hline \multicolumn{3}{|l|}{ Infarct location, $n(\%)$} & \multirow[t]{6}{*}{0.149} \\
\hline Anterior wall & $140(45.9)$ & $36(64.3)$ & \\
\hline Inferior wall & $55(18)$ & $6(10.7)$ & \\
\hline Inferior and posterior wall & $43(14.1)$ & $4(7.1)$ & \\
\hline Complicate by right ventricular & $63(20.7)$ & $10(17.9)$ & \\
\hline Others & $4(1.3)$ & $0(0)$ & \\
\hline \multicolumn{4}{|l|}{ Aspiration thrombectomy } \\
\hline Yes & $206(67.5)$ & $40(71.4)$ & \multirow[t]{2}{*}{0.566} \\
\hline No & $99(32.5)$ & $16(28.6)$ & \\
\hline \multicolumn{4}{|l|}{ Upfront intracoronary GPIIb/IIIa receptor inhibitor } \\
\hline Yes & $48(15.7)$ & $3(5.4)$ & \multirow[t]{2}{*}{$0.040^{*}$} \\
\hline No & $257(84.3)$ & $53(94.6)$ & \\
\hline \multicolumn{4}{|l|}{ Non-IRA intervention, $n(\%)$} \\
\hline Yes & $18(5.9)$ & $4(7.1)$ & \multirow[t]{2}{*}{0.760} \\
\hline No & $287(94.1)$ & $52(92.9)$ & \\
\hline
\end{tabular}

${ }^{*} P<0.05$.

had significantly higher neutrophil counts than those in the normal reflow group. In univariate and multivariate logistic regression analyses, a neutrophil count above $9.14 \times 10^{9} / \mathrm{L}$ was independently associated with no-reflow after adjusting for age $\geq 65$, male, smoking history, hypertension, diabetes, Killip classification $\geq 3$, LAD as the IRA, neutrophil/lymphocyte ratio, cTNI, upfront intracoronary GPIIb/IIIa inhibitor administration, aspiration thrombectomy, platelet counts, WBC counts, HGB, time from symptom onset to reperfusion ( $>6$ hours), multivessel disease, and initial TIMI flow grade $(0-1)$.

The present result is supported by the retrospective study of Kosuge et al. [18] stating that a WBC count of 12,000 cells/ $\mathrm{mm}^{3}$ was an independent predictor of impaired myocardial reperfusion in patients with early recanalized anterior acute myocardial infarction (AMI). However, they only recruited the patients with anterior AMI. Furthermore, they did not analyse specific types of WBCs. Takahashi et al. [19] concluded that neutrophils over $10 \mathrm{G} / \mathrm{L}$ were associated with no reflow within 116 patients with a first anterior AMI. The present study recruited patients not limited to those with anterior AMI. The study of Wang et al. [20] which assessed the relationship between neutrophil counts and no-reflow included 217 patients in the author's center, age and blood cells counts were adjusted exclusively in the study, and the threshold of neutrophil count no-reflow was not analysed. In the present study, we obtained a cut-off value of $9.14 \times 10^{9} / \mathrm{L}(67.9 \%$ sensitivity and $57.7 \%$ specificity), with neutrophil counts above this threshold being associated with increased rates of no-reflow. There were studies on the prognostic value of neutrophil to lymphocyte ratio in predicting no-reflow $[15,16]$; hence, the present study also included neutrophil/lymphocyte ratio in the multivariate analysis. Additionally, other factors that are clinically relevant to noreflow including hypertension, blood pressure, Killip classification $(\geqq 3)$, cTNI, time from symptom onset to reperfusion ( $>6$ hours), multivessel disease, and initial TIMI flow grade $(0-1)$ were also included in the multivariate analysis in the 
TABLE 5: Univariate and multivariate logistic analysis for no-reflow.

\begin{tabular}{|c|c|c|c|c|c|c|}
\hline \multirow{2}{*}{ Parameters } & \multicolumn{3}{|c|}{ Univariate logistic analysis } & \multicolumn{3}{|c|}{ Multivariate logistic analysis } \\
\hline & OR & $95 \%$ CI & $P$ & OR & $95 \% \mathrm{CI}$ & $P$ \\
\hline Age $\geq 65$ years & 1.116 & $0.567-2.199$ & 0.751 & 1.068 & $0.467-2.442$ & 0.877 \\
\hline Male & 1.115 & $0.446-2.786$ & 0.815 & 1.364 & $0.441-4.224$ & 0.590 \\
\hline Smoking & 1.171 & $0.640-2.143$ & 0.610 & 1.148 & $0.549-2.400$ & 0.714 \\
\hline Hypertension & 1.021 & $0.577-1.809$ & 0.942 & 0.979 & $0.519-1.845$ & 0.947 \\
\hline Diabetes & 0.938 & $0.486-1.807$ & 0.847 & 0.821 & $0.395-1.710$ & 0.599 \\
\hline Killip classification $(\geqq 3)$ & 2.824 & $1.155-6.904$ & $0.023^{*}$ & 4.072 & $1.391-11.916$ & $0.010^{*}$ \\
\hline LAD as IRA & 1.821 & $1.018-3.259$ & $0.043^{*}$ & 2.457 & $1.226-4.925$ & $0.011^{*}$ \\
\hline Neutrophil count $\geq 9.14 \times 10^{9} / \mathrm{L}$ & 2.880 & $1.573-5.275$ & $0.001^{* *}$ & 4.474 & $1.610-12.433$ & $0.004^{* *}$ \\
\hline Neutrophil/lymphocyte ratio & 1.067 & $1.015-1.121$ & $0.011^{*}$ & 1.029 & $0.967-1.095$ & 0.366 \\
\hline cTNI & 1.004 & $1.000-1.008$ & $0.036^{*}$ & 1.001 & $0.995-1.006$ & 0.806 \\
\hline Upfront intracoronary GPIIb/IIIa receptor inhibitor & 0.303 & $0.091-1.010$ & 0.052 & 0.219 & $0.061-0.783$ & $0.019^{*}$ \\
\hline Aspiration thrombectomy & 1.201 & $0.642-2.250$ & 0.566 & 1.253 & $0.469-3.347$ & 0.652 \\
\hline Platelet counts & 0.998 & $0.993-1.003$ & 0.424 & 0.997 & $0.991-1.003$ & 0.343 \\
\hline WBC counts $\left(\times 10^{9} / \mathrm{L}\right)$ & 1.086 & $1.002-1.178$ & $0.046^{*}$ & 0.940 & $0.798-1.107$ & 0.459 \\
\hline HGB (g/L) & 0.992 & $0.973-1.011$ & 0.390 & 0.982 & $0.959-1.007$ & 0.159 \\
\hline Time from symptoms onset to reperfusion ( $>6$ hours) & 0.871 & $0.465-1.632$ & 0.666 & 0.932 & $0.469-1.851$ & 0.840 \\
\hline Multivessel disease & 1.004 & $0.520-1.940$ & 0.990 & 0.987 & $0.470-2.075$ & 0.973 \\
\hline Initial TIMI flow grade $(0-1)$ & 1.242 & $0.677-2.280$ & 0.483 & 0.886 & $0.354-2.219$ & 0.796 \\
\hline
\end{tabular}

${ }^{*} P<0.05 ;{ }^{* *} P<0.01$. Age $\geq 65$ years, male, smoking, hypertension, diabetes, Killip classification ( $\left.\geqq 3\right)$, LAD as IRA, neutrophil count $\geq 9.14 \times 10^{9} / \mathrm{L}$, neutrophil/ lymphocyte ratio, cTNI, upfront intracoronary GPIIb/IIIa receptor inhibitor, aspiration thrombectomy, platelet counts, WBC counts, HGB, time from symptoms onset to reperfusion ( $>6$ hours), multivessel disease, and initial TIMI flow grade (0-1) were included in the multivariate analysis.

present study. The prognostic association of the neutrophil/ lymphocyte ratio with no-reflow was lost after adjusting for neutrophil count $\geq 9.14 \times 10^{9} / \mathrm{L}$. Furthermore, neutrophil/ lymphocyte ratio was not associated with no-reflow in multivariate analysis without confounding for neutrophil counts $(\mathrm{OR}=1.043$, 95\%CI: $0.984-1.106, P=0.152)$. This could be explained by the elevated neutrophil/lymphocyte ratio in our study that mainly resulted from the increased neutrophil counts.

The underlying mechanism of the involvement of neutrophils in no-reflow is complex. Ischemic injury damages myocardiocytes, which presents as myocardial cell swelling and interstitial edema. The pathological changes in myocardial cells increase the compression of intramural vessels and induce neutrophil plugging and activation in the coronary microcirculation [4]. The oxygen-free radicals released by activated neutrophils contribute to endothelial injury and impaired reperfusion. At the time of reperfusion, there was a massive neutrophil adhesion to the endothelium due to the excessive generation of reactive oxygen species and subsequently activated NF- $\kappa$ B cascade. Structural luminal obstruction of the microvasculature resulted from microaggregates formed by neutrophils and platelets that aggravate the reperfusion injury $[4,21]$. Moreover, intense and prolonged coronary microvascular vasoconstriction is attributable to vasoactive substances produced by neutrophils, platelets, and damaged endothelial cells [11]. In addition, the infiltration of neutrophils in the vulnerable myocardium as a result of increased vascular permeability enhances interstitial edema and extravascular mechanical compression, contributing to the pathological processes of no-reflow $[4,11]$.

ACS is a group of clinical syndromes characterized by rupture or erosion of coronary atherosclerotic plaques and subsequent complete or incomplete thrombotic occlusion [22]. Lipid-rich plaques are correlated with impaired reperfusion after restoration of the epicardial artery [23]. Noncalcified plaque burden is correlated with the neutrophil/lymphocyte ratio [24]. Neutrophils mediate apoptosis in endothelial cells and smooth muscle cells, contributing to plaque rupture [25-27]. Microvascular embolization and no-reflow occur when a mass of plaque fragments, leading to the release of cholesterol crystals and microthrombi into the bloodstream. Moreover, active neutrophils accelerate the formation of platelet-leukocyte aggregates, plugging the microvessel.

In the present study, we found that the Killip classification $\geq 3$ was associated with no-reflow. Patients with no-reflow had advanced Killip classifications, coincident with the findings of Zhou et al. [17], despite that Killip classification 2 rate in the present was higher than that reported. There were $7.9 \%$ patients with Killip classifications 3 and 4, similar to the percentage in the study of Zhou et al. (8.9\%) [17]. Killip classification of at least grade 3 on admission may be associated with larger infarctions and decreased coronary perfusion pressure [17]. The decreased coronary pressure accelerates plugging of neutrophils in the microvasculature, inducing no-reflow. Although 
we observed the cTNI (associated with larger infarct area) level was higher in group $B(<9.4 \mathrm{G} / \mathrm{L})$ than in group $\mathrm{A}(\geq 9.4 \mathrm{G} / \mathrm{L})$, there was no difference between the no-reflow group and normal reflow group. Furthermore, after adjusting for cTNI, neutrophil count was independently associated with no-reflow.

4.1. Limitations. There are some limitations to the present study. A relatively small sample size was used. Furthermore, only TIMI flow grade was used to identify no-reflow, and no other diagnostic methods were used because of limited data. This partially explains why the LAD as the IRA was a negative factor for no-reflow. However, this difference did not affect the outcomes of the study. A prospective study including a larger sample, and in which TIMI myocardial perfusion or myocardial blush grade is assessed, is needed in the future.

\section{Conclusions}

Inflammatory responses and the infiltration of neutrophils are associated with ischemia/reperfusion injury associated with no-reflow following PCI. A circulating neutrophil count $\geq 9.14 \times 10^{9} / \mathrm{L}$ is independently associated with noreflow in patients with acute STEMI following successful primary PCI.

\section{Conflicts of Interest}

The authors declare that they have no conflicts of interest.

\section{Authors' Contributions}

Shuzheng Lyu contributed to the topic conception and manuscript revision. Jinfan Tian participated in the study design, data collection, data analysis and interpretation, and writing of the manuscript. Yue Liu contributed to data analysis, writing, and revision of manuscript and is the co-first author. Yanfei Liu contributed to data collection and analysis. Xiantao Song, Min Zhang, Feng $\mathrm{Xu}$, and Fei Yuan participated in the decision for treatment strategies, data collection, and analysis. Jinfan Tian and Yue Liu contributed equally to this work.

\section{Acknowledgments}

The authors gratefully acknowledge the financial support from the Beijing NOVA Program (no. Z171100001117027), Key Projects in the National Science and Technology Pillar Program during the 12th Five-Year Plan Period (no. 2011BAI11B05), and Beijing Lab for Cardiovascular Precision Medicine (PXM2017_014226_000037). The authors also thank Jing Dai, Wei Wang, Dongfeng Zhang, and Mingduo Zhang, who helped in recruiting the patients, and the patients who agreed to participate in this trial.

\section{References}

[1] R. A. Kloner, C. E. Ganote, and R. B. Jennings, "The "noreflow" phenomenon after temporary coronary occlusion in the dog," The Journal of Clinical Investigation, vol. 54, no. 6, pp. 1496-1508, 1974.
[2] G. Galasso, S. Schiekofer, C. D'Anna et al., "No-reflow phenomenon: pathophysiology, diagnosis, prevention, and treatment. A review of the current literature and future perspectives," Angiology, vol. 65, no. 3, pp. 180-189, 2014.

[3] G. Ndrepepa, K. Tiroch, M. Fusaro et al., "5-year prognostic value of no-reflow phenomenon after percutaneous coronary intervention in patients with acute myocardial infarction," Journal of the American College of Cardiology, vol. 55, no. 21, pp. 2383-2389, 2010.

[4] A. Durante and P. G. Camici, "Novel insights into an "old" phenomenon: the no reflow," International Journal of Cardiology, vol. 187, pp. 273-280, 2015.

[5] W. J. van Gaal and A. P. Banning, "Percutaneous coronary intervention and the no-reflow phenomenon," Expert Review of Cardiovascular Therapy, vol. 5, no. 4, pp. 715-731, 2007.

[6] B. Chandrasekar, J. B. Smith, and G. L. Freeman, "Ischemiareperfusion of rat myocardium activates nuclear factor- $\kappa \mathrm{B}$ and induces neutrophil infiltration via lipopolysaccharideinduced CXC chemokine," Circulation, vol. 103, no. 18, pp. 2296-2302, 2001.

[7] L. Ge, X. Zhou, W. J. Ji et al., "Neutrophil extracellular traps in ischemia-reperfusion injury-induced myocardial no-reflow: therapeutic potential of DNase-based reperfusion strategy," American Journal of Physiology Heart and Circulatory Physiology, vol. 308, no. 5, pp. H500-H509, 2015.

[8] M. Zeng, H. Yan, Y. Chen et al., "Suppression of NF- $\kappa \mathrm{B}$ reduces myocardial no-reflow," PLoS One, vol. 7, no. 10, article e47306, 2012.

[9] S. A. Briaud, Z. M. Ding, L. H. Michael, M. L. Entman, S. Daniel, and C. M. Ballantyne, "Leukocyte trafficking and myocardial reperfusion injury in ICAM-1/P-selectin-knockout mice," American Journal of Physiology Heart and Circulatory Physiology, vol. 280, no. 1, pp. H60-H67, 2001.

[10] K. R. Martin, D. Ohayon, and V. Witko-Sarsat, "Promoting apoptosis of neutrophils and phagocytosis by macrophages: novel strategies in the resolution of inflammation," Swiss Medical Weekly, vol. 145, article w14056, 2015.

[11] D. T. Wong, R. Puri, J. D. Richardson, M. I. Worthley, and S. G. Worthley, "Myocardial "no-reflow" - diagnosis, pathophysiology and treatment," International Journal of Cardiology, vol. 167, no. 5, pp. 1798-1806, 2013.

[12] L. Ao, N. Zou, J. C. Cleveland Jr., D. A. Fullerton, and X. Meng, "Myocardial TLR4 is a determinant of neutrophil infiltration after global myocardial ischemia: mediating KC and MCP-1 expression induced by extracellular HSC70," American Journal of Physiology Heart and Circulatory Physiology, vol. 297, no. 1, pp. H21-H28, 2009.

[13] K. Distelmaier, M. P. Winter, F. Dragschitz et al., "Prognostic value of culprit site neutrophils in acute coronary syndrome," European Journal of Clinical Investigation, vol. 44, no. 3, pp. 257-265, 2014.

[14] P. G. Steg, S. K. James, D. Atar et al., "ESC guidelines for the management of acute myocardial infarction in patients presenting with ST-segment elevation: the Task Force on the management of ST-segment elevation acute myocardial infarction of the European Society of Cardiology (ESC)," European Heart Journal, vol. 33, no. 20, pp. 2569-2619, 2012.

[15] S. Wagdy, M. Sobhy, and M. Loutfi, "Neutrophil/lymphocyte ratio as a predictor of in-hospital major adverse cardiac events, new-onset atrial fibrillation, and no-reflow phenomenon in patients with ST elevation myocardial infarction," Clinical Medicine Insights: Cardiology, vol. 10, pp. 19-22, 2016. 
[16] S. Turkmen, O. Dogdu, K. Tekin et al., "The relationship between neutrophil/lymphocyte ratio and the TIMI flow grade in patients with STEMI undergoing primary PCI," European Review for Medical and Pharmacological Sciences, vol. 17, no. 16, pp. 2185-2189, 2013.

[17] H. Zhou, X. Y. He, S. W. Zhuang et al., "Clinical and procedural predictors of no-reflow in patients with acute myocardial infarction after primary percutaneous coronary intervention," World Journal of Emergency Medicine, vol. 5, no. 2, pp. 96-102, 2014.

[18] M. Kosuge, K. Kimura, T. Ishikawa et al., "Relation between white blood cell counts and myocardial reperfusion in patients with recanalized anterior acute myocardial infarction," Circulation Journal, vol. 68, no. 6, pp. 526-531, 2004.

[19] T. Takahashi, Y. Hiasa, Y. Ohara et al., "Relation between neutrophil counts on admission, microvascular injury, and left ventricularfunctional recovery in patients with an anterior wall first acute myocardial infarction treated with primary coronary angioplasty," The American Journal of Cardiology, vol. 100, no. 1, pp. 35-40, 2007.

[20] Z. Wang, L. Ren, L. Lei, H. Ye, and J. Peng, "The relationship between neutrophil counts on admission and angiographic no-reflow after primary percutaneous coronary intervention in patients with ST-segment elevation myocardial infarction," Acta Cardiologica, vol. 71, no. 2, pp. 241-246, 2016.

[21] D. J. Hausenloy and D. M. Yellon, "Targeting myocardial reperfusion injury-the search continues," The New England Journal of Medicine, vol. 373, no. 11, pp. 1073-1075, 2015.

[22] P. Libby, "Mechanisms of acute coronary syndromes and their implications for therapy," The New England Journal of Medicine, vol. 368, no. 21, pp. 2004-2013, 2013.

[23] J. Li, L. Wu, X. Tian, J. Zhang, and Y. Shi, "Intravascular ultrasound observation of the mechanism of no-reflow phenomenon in acute myocardial infarction," PLoS One, vol. 10, no. 6, article e119223, 2015.

[24] L. Nilsson, W. G. Wieringa, G. Pundziute et al., "Neutrophil/ lymphocyte ratio is associated with non-calcified plaque burden in patients with coronary artery disease," PLoS One, vol. 9, no. 9, article e108183, 2014.

[25] M. Madjid, I. Awan, J. T. Willerson, and S. W. Casscells, "Leukocyte count and coronary heart disease: implications for risk assessment," Journal of the American College of Cardiology, vol. 44, no. 10, pp. 1945-1956, 2004.

[26] P. Libby, M. Nahrendorf, and F. K. Swirski, "Leukocytes link local and systemic inflammation in ischemic cardiovascular disease: an expanded "cardiovascular continuum"," Journal of the American College of Cardiology, vol. 67, no. 9, pp. 1091-1103, 2016.

[27] P. Rotzius, S. Thams, O. Soehnlein et al., "Distinct infiltration of neutrophils in lesion shoulders in $\mathrm{ApoE}^{-/-}$mice," The American Journal of Pathology, vol. 177, no. 1, pp. 493-500, 2010. 


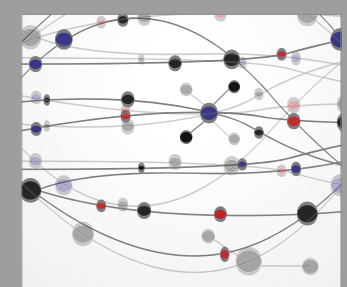

The Scientific World Journal
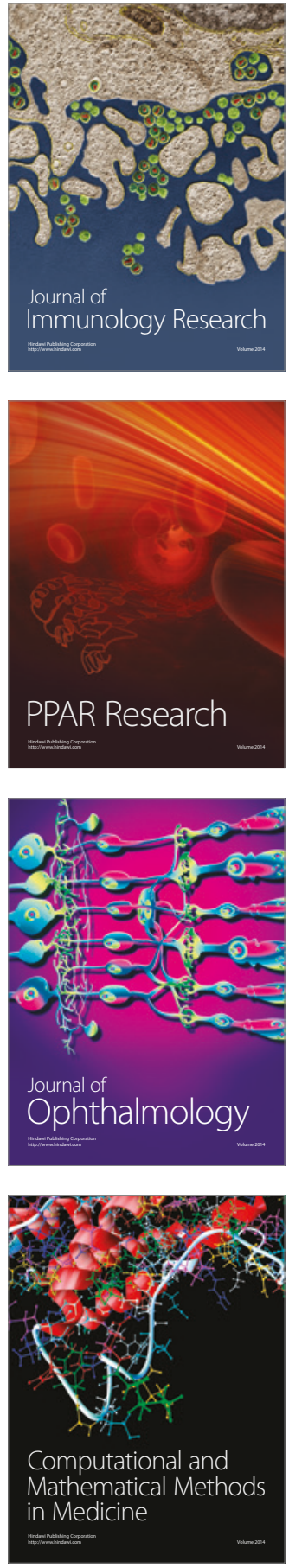

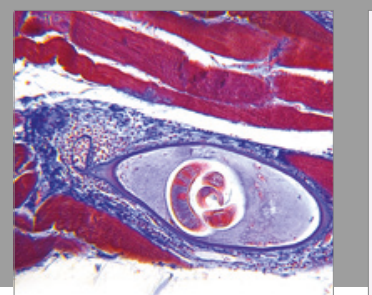

Gastroenterology Research and Practice
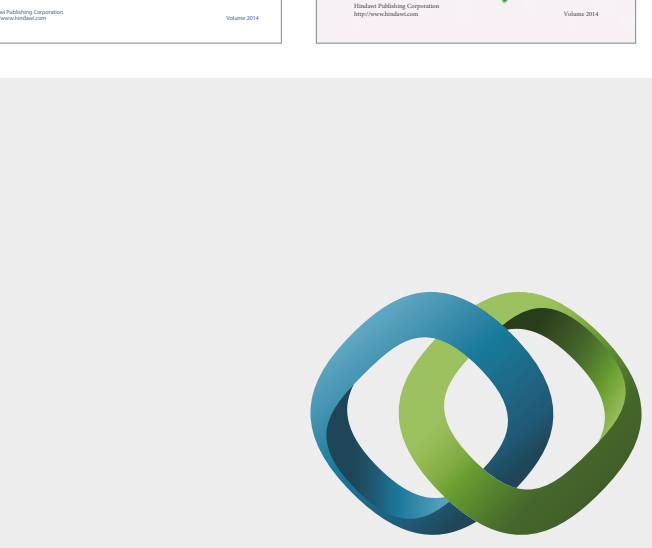

\section{Hindawi}

Submit your manuscripts at

https://www.hindawi.com
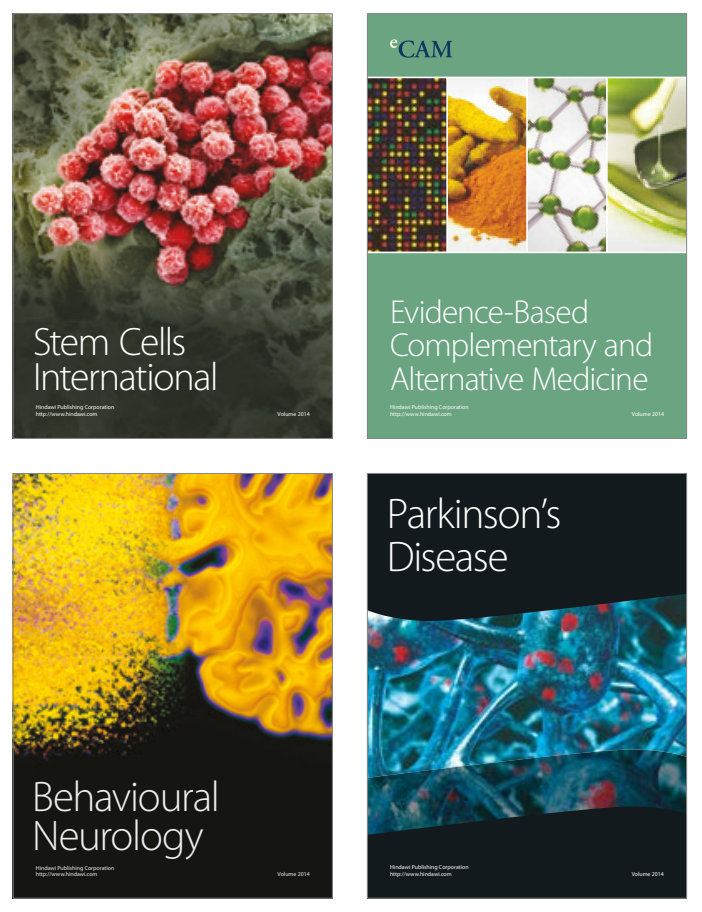
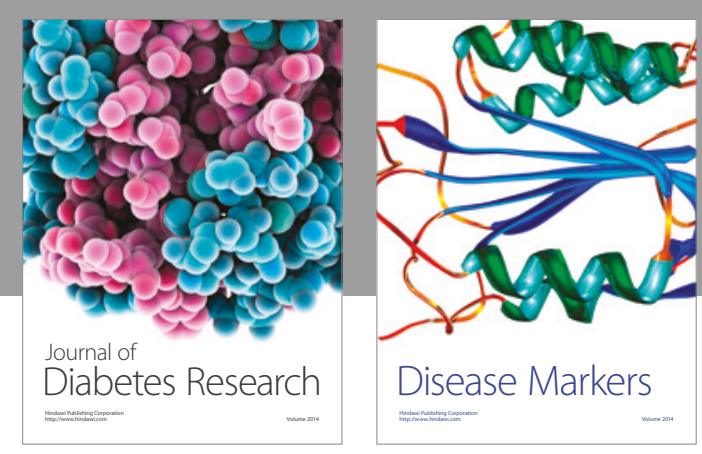

Disease Markers
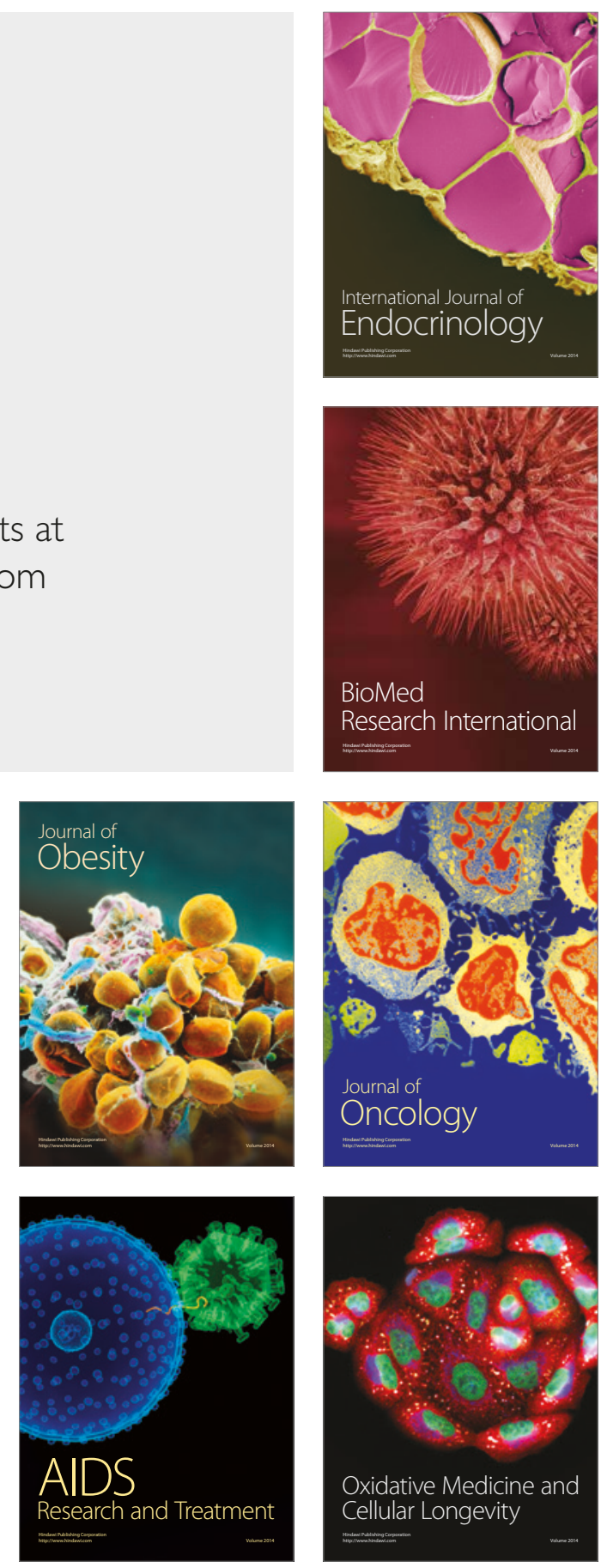